\title{
THE ACUTE EFFECTS OF DIFFERENT RESISTANCE TRAINING LOADS ON REPEATED SPRINT ABILITY IN WATER POLO PLAYERS
}

original paper

(c) University School of Physical Education in Wroclaw

DOI: https://doi.org/10.5114/hm.2021.103293

\author{
ATHANASIOS A. DALAMITROS ${ }^{1}$, PANAGIOTIS OROLOGAS ${ }^{2}$, STAMATIA NOUSIOU ${ }^{1}$, \\ ELENI SEMALTIANOU ${ }^{1}$, ATHANASIA ZOURLADANI ${ }^{1}$, VASILIKI MANOU ${ }^{1}$ \\ ${ }^{1}$ Laboratory of Evaluation of Human Biological Performance, School of Physical Education and Sports Sciences, \\ Aristotle University of Thessaloniki, Thessaloniki, Greece \\ ${ }^{2}$ School of Physical Education and Sports Sciences, Democritus University of Thrace, Komotini, Greece
}

\section{ABSTRACT}

Purpose. Combining resistance and in-water training is a common practice in water polo. The purpose of this study was to examine the acute effects of different external loads during resistance training on the repeated sprint ability in water polo players.

Methods. A total of 9 national level water polo players (age: $21.5 \pm 2.0$ years) executed, on different occasions, a repeated sprint swimming test $(8 \times 25 \mathrm{~m}$ with a 30 -s rest) following a low load $(50 \%$ of 1 -repetition maximum) or a high load $(80 \%$ of 1-repetition maximum) resistance training that consisted of leg press and bench press exercises. During the control condition, the players only performed the repeated ability test. Indices of repeated sprint ability included the best $25-\mathrm{m}$ time (best), the mean time (mean), and the heart rate after the $4^{\text {th }}$ and the $8^{\text {th }} 25-\mathrm{m}$ effort. In addition, the performance decrement was calculated.

Results. Control and low load intensity conditions resulted in significantly faster best times than high load intensity $(p<0.01$ and 0.05 , respectively). Mean times were significantly faster during the control compared with the high load intensity condition $(p<0.05)$. Performance decrement and heart rate after the $4^{\text {th }}$ and the $8^{\text {th }} 25-\mathrm{m}$ effort showed no differences between all 3 conditions $(p>0.05)$.

Conclusions. These results suggest that when scheduling intrasession resistance and in-water training in water polo, low load programs should be preferred when the primary goal is the enhancement of repeated swimming performance.

Key words: concurrent training, different training intensities, sprint performance, water polo

\section{Introduction}

Combining resistance training (RT) and high-intensity efforts, as a variation of the training modality refereed to as concurrent training (i.e., the simultaneous combination of RT and endurance training) [1], is suggested as a time-efficient method that can minimize the potential adverse effects of RT adaptations $[2,3]$ attributed to the so-called interference phenomenon [4]. Indeed, a recent review and meta-analysis [5] stated that, under certain conditions (e.g., adequate rest interval, selection of predominant muscle groups utilized during interval sets), concurrent RT and high- intensity training can be prescribed without compromising any strength gains.

The ability to perform during repeated maximal sprint efforts - repeated sprint ability (RSA), a category of high-intensity training, is of great importance in water polo considering its intermittent high-intensity nature [6, 7]. Along with the fact that muscle strength is a prerequisite for success in water polo [8], it can be postulated that concurrent RT and RSA programs may have an important practical application in this sport. As such, Botonis et al. [9] examined longterm effects (27 weeks) of a concurrent RT and highintensity interval program $(4 \times 4$-minute swimming),

Correspondence address: Athanasios A. Dalamitros, Aristotle University of Thessaloniki, School of Physical Education and Sports Sciences, Thermi Thessalonikis, 57001, Thessaloniki, Greece, e-mail: dalammi@phed.auth.gr

Received: June 23, 2020

Accepted for publication: August 6, 2020

Citation: Dalamitros AA, Orologas P, Nousiou S, Semaltianou E, Zourladani A, Manou V. The acute effects of different resistance training loads on repeated sprint ability in water polo players. Hum Mov. 2021;22(4):78-82; doi: https://doi.org/10.5114/ hm.2021.103293. 
including specific water polo training, on RSA (8 repetitions of 20-m maximal sprints), as well as other exercise performance variables and match-induced fatigue indices. In this study, the maintenance of RSA throughout the tested season was reported.

Among a large number of variables (e.g., inter-individual responses, exercise volume and duration, training background, type and sequence of exercise, etc.) $[3,10,11]$ related to both acute and chronic mechanisms [12], training intensity is considered as one of the most important ones affecting the magnitude of interference during concurrent training programs design [13]. In all the above-mentioned studies, the examined variables were related to strength (muscle hypertrophy, power) and endurance adaptations (kinematics, energy expenditure, performance indices, etc). To our knowledge, there are no respective data regarding RSA performance.

As, in real conditions, a great part of training in water polo usually combines intrasession RT and RSA efforts [14], it would be interesting to analyse the acute effect of a concurrent training program on RSA indices after different RT intensities in water polo players. Thus, our purpose was to compare the acute effects of 2 RT programs, differed in the load intensity, on RSA indices, namely maximal swim time, mean swim time, and performance decrement (PD). It was hypothesized that greater RT load intensities would result in a more profound reduction in the subsequent RSA performance.

\section{Material and methods}

\section{Participants}

Overall, 9 national level male water polo players (age: $21.5 \pm 2.0$ years, body height: $181.6 \pm 8.0 \mathrm{~cm}$, body mass: $75.2 \pm 10.9 \mathrm{~kg}$, training experience: $11.6 \pm$ 1.2 years) volunteered to participate in this study. All subjects were competitive water polo players, members of the same team, and trained 5-6 times per week, including twice-weekly RT programs. All participants were free from musculoskeletal disorders by the time this study was conducted. No use of supplements was reported.

\section{Procedures}

The participants were engaged in 4 testing days. On day 1, anthropometric (body height and weight) and training (training experience) characteristics were recorded, along with the 1-repetition maximum (1RM) test on the bench press and leg press exercises to quan- tify the different RT load intensities. During the remaining 3 days, the subjects were randomly assigned into 3 different testing conditions: the low load RT intensity (LLT), the high load RT intensity (HLT), and the control condition (CON). In CON, the participants only executed the RSA test. All testing days were separated by 48 hours. All athletes completed the 3 testing conditions at the same time of the day, while effort was made to follow the same training routine the day before testing. A period of approximately 20 minutes was employed between each RT program and RSA testing.

\section{Resistance training program}

Both RT programs included 3 sets of 8 repetitions on the bench press and leg press exercises, with a 2 -minute rest between sets and a 3-minute rest between exercises. The LLT program was executed with the intensity corresponding to $50 \%$ of $1 \mathrm{RM}$, while the respective intensity for the HLT program was $80 \%$ of $1 \mathrm{RM}$. The participants were instructed to execute LLT on a high movement velocity. All RT programs were supervised by the research associates.

\section{Repeated sprint ability test}

After completing a standardized in-water warm-up consisting of 500-m easy swim, drills, and progressively faster swims, as well as a 2-minute interval, the participants executed the RSA test. The test included 8 repetitions of a single $25-\mathrm{m}$ all-out sprint set $(8 \times$ $25 \mathrm{~m}$ ), executed with the front-crawl technique. Each 25-m effort was separated by a 30-second rest interval. The players started each sprint with a push-start, while they were instructed to start swimming immediately after their feet left the wall [15]. Indices of RSA included the best $25-\mathrm{m}$ time (best), the mean time during the RSA test (mean), and PD, calculated as previously suggested [16]. Exercise heart rate was recorded immediately after the $4^{\text {th }}\left(\mathrm{HR}^{4 \text { th }}\right)$ and the $8^{\text {th }}\left(\mathrm{HR}^{8 \text { th }}\right)$ sprint effort, by using chest belt telemetry (Polar Electro, Kempele, Finland). Two experienced researchers timed each 25-m effort individually with a commercial stopwatch (Seiko S141, Japan). The mean value was used for further analysis. No feedback related to RSA performance was given to the participants until the completion of all 3 conditions [17].

\section{Statistical analysis}

Data are reported as means \pm standard deviations. All statistical tests were processed with the use of the 
A. Dalamitros et al., Intrasession resistance and sprint training in water polo

SPSS statistical package (v. 21; SPSS Inc., Chicago, USA). Sphericity was verified by Mauchly's test. When the assumption of sphericity was not met, the significance of $F$-ratios was adjusted in accordance with the Greenhouse-Geisser procedure. Repeated measures analysis of variance was applied to compare best, mean, $\mathrm{PD}, \mathrm{HR}^{4 \text { th }}$, and $\mathrm{HR}^{\text {th }}$ during the 3 different conditions. Paired samples $t$-tests with a Bonferroni correction were used to analyse between-condition differences. Cohen's $d$ effect sizes ( $d=$ difference between means / pooled standard deviation) were calculated for the difference between the means. The small, medium, and large effects were reflected in values greater than 0.20 , 0.50 , and 0.80 , respectively [18]. Statistical significance of the results was set as $p<0.05$.

\section{Ethical approval}

The research related to human use has complied with all the relevant national regulations and institutional policies, has followed the tenets of the Declaration of Helsinki, and has been approved by the authors' institutional review board (approval No.: EC-6/2020).

\section{Informed consent}

Informed consent has been obtained from all individuals included in this study.

\section{Results}

The results of the repeated measures ANOVA revealed a significant difference between conditions regarding the single best $25-\mathrm{m}$ effort and mean times $\left(F_{2}=8.88, p=0.003\right.$ and $F_{2}=4.33, p=0.031$, respectively). PD and both HR measurements ( $\mathrm{HR}^{4 \text { th }}$ and $\left.\mathrm{HR}^{8 \mathrm{sth}}\right)$ were not different between the conditions $\left(F_{2}=\right.$ 1.94 to 3.29 , and $p=0.06$ to 0.177 ) (Table 1 ). With refer- ence to paired samples $t$-tests, best times were faster during CON and LLT compared with the HLT condition ( $p=0.007$ and 0.016 , respectively; $d=0.51$ to 0.60 [medium effect]). No difference was shown between CON and LLT ( $p=0.519, d=0.10$ [small effect]). Regarding mean times, enhanced performance was observed during CON compared with HLT ( $p=0.025$, $d=0.32$ [small effect]), but not LLT ( $p=0.127, d=0.29$ [small effect]. Similarly, no difference was shown between the LLT and HLT conditions ( $p=0.261, d=0.01$ [small effect]). No differences were noticed between the rest of the variables analysed $\left(\mathrm{PD}, \mathrm{HR}^{4 \mathrm{th}}\right.$, and $\mathrm{HR}^{8 \mathrm{th}}$ ) $(p>0.05)$ (Table 1).

\section{Discussion}

Following our hypothesis, the participants exhibited greater reductions in RSA performance after the execution of the HLT protocol compared with the LLT protocol and the control condition. However, this reduction was not significant for all indices of RSA analysed, as PD was almost similar between the different conditions.

Although the combination of RT and high-interval efforts during a single training session has been extensively analysed for parameters related to muscular strength [5], to the authors' knowledge, this is the first study focused on examining the acute effects of $2 \mathrm{RT}$ protocols differing in relative intensity (\%1RM) on the indices evaluated during sprint-interval training, and specifically in water polo players. A previous training study that included different RT external loads and continuous endurance training reported almost similar (low to moderate) improvements in maximal sprint efforts in 10-30-m distances in a physically active population [11].

Table 1. Mean \pm standard deviations and $p$ values for repeated sprint ability indices in the 3 different conditions

\begin{tabular}{lcccc}
\hline \multirow{2}{*}{ Variable } & \multicolumn{3}{c}{ Testing condition } & \multicolumn{1}{c}{$p$} \\
\cline { 2 - 4 } & LLT & HLT & CON & $0.003^{* *}$ \\
\hline Best (s) & $13.77 \pm 0.74$ & $14.16 \pm 0.78$ & $13.70 \pm 0.76$ \\
Mean (s) & $14.87 \pm 0.74$ & $14.76 \pm 0.84$ & $14.50 \pm 0.80$ & $0.031^{*}$ \\
PD (\%) & $10.94 \pm 0.89$ & $10.89 \pm 1.91$ & $9.14 \pm 2.13$ & 0.154 \\
HR $^{4 \text { th }}$ (beats $\cdot \mathrm{min}^{-1}$ ) & $174.4 \pm 5.2$ & $176.4 \pm 6.7$ & $170.0 \pm 9.8$ & 0.064 \\
HR $^{\text {th }}$ (beats $\cdot \mathrm{min}^{-1}$ ) & $176.4 \pm 7.0$ & $181.2 \pm 9.1$ & $177.1 \pm 12.2$ & 0.177 \\
\hline
\end{tabular}

LLT - low load intensity resistance training (50\% 1-repetition maximum) followed by the repeated sprint ability test, HLT - high load intensity resistance training (80\% 1-repetition maximum) followed by the repeated sprint ability test, $\mathrm{CON}$ - repeated sprint ability test with no prior resistance training,

Best - best 25-m effort time, Mean - mean $8 \times 25$-m time, PD - performance decrement during the repeated sprint ability test, $\mathrm{HR}^{4 \text { th }}$ - heart rate immediately after the $4^{\text {th }} 25$-m effort, $\mathrm{HR}^{8 \text { th }}$ - heart rate immediately after the $8^{\text {th }} 25$-m effort

${ }^{*} p<0.05,{ }^{*} p<0.01$ between the 3 different conditions 
Combining strength and in-water training is a timeefficient and common practice in water polo. In this full-contact sport, high levels of muscular strength are essential for water polo athletes [8, 19]. Moreover, muscular strength is also positively correlated with swimming velocity during short distances [20], while repeated short sprints are frequently used in water polo to mimic game situations [21]. The selected exercises (bench press and leg press) that were applied during the RT program had been also used in previous water polo studies [8, 14]. The criterion for the RT exercise selection was the activation of particular muscle groups mostly used in water polo [9].

Since the training sequence is a significant parameter when designing concurrent training programs [22], RT was conducted prior to the in-water training in both LLT and HLT conditions in this study. Furthermore, an effort was made to control as many interindividual parameters as possible (i.e., recovery time between RT and RSA test, chronological age and gender, participants' competitive and strength level, RT intrasession exercise sequence). As none of the indices evaluated during the RSA test was previously correlated with the aerobic fitness in water polo athletes [15], no attempt to include the respective training parameter was made here.

The participants in this study were members of a second division team of the national championship, which explains the slower single best $25-\mathrm{m}$ performance time reported compared with the study by Meckel et al. [15], involving elite water polo athletes (13.70 vs. 12.26 s, respectively). However, similar comparisons regarding the rest of the indices analysed cannot be conducted since different protocols during the RSA test were used in previous water polo studies. The observed significant differences between the best 25-m effort and mean RSA times between the CON and the HLT, but not the LLT RT condition can be attributed to the possible appearance of residual fatigue, as higher intensities during RT were implemented ( $80 \%$ vs. $50 \%$ of $1 \mathrm{RM}$ in this study); thus, higher threshold motor units were recruited similarly with the demands of the high-intensity effort applied [5] in terms of the RSA test. This interference effect could probably not be diminished during the $20-\mathrm{m}$ period interpolated between the RT and the in-water test. PD showed an almost similar trend between the conditions. Apparently, the relatively low repetitions used during the RSA test $(8 \times 25 \mathrm{~m})$ or the work:rest ratio (almost 1:2) led to this result. The fact that $\mathrm{HR}^{4 \text { th }}$ and $\mathrm{HR}^{8 \mathrm{sth}}$ values were not different between the 3 condi- tions may indicate that all swim efforts were executed with equal intensity.

An obvious limitation of the current study is the lack of an electronic time recording system regarding the 25-m maximum performance times. Even so, the 2 research collaborators that were recruited for the task were experienced and certified timekeepers, while caution was given to assess the same participants in each pool condition. This methodology, however, has been used previously in swimming studies. Finally, it seems reasonable to emphasize the low number of participants. In our initial research design, at least 3 more athletes were included; however, the restricted measures imposed during the COVID-19 pandemic were an obstacle in this research.

\section{Conclusions}

In conclusion, the results presented here seem to suggest that when scheduling intrasession resistance and in-water training in water polo, relatively low external loads should be preferred when the primary goal is the improvement of repeated sprint swimming performance. Similar studies should also consider the longitudinal effects of such training designs on both strength gains and sprint performance in water polo.

\section{Acknowledgements}

The authors would like to thank the participants for their contribution.

\section{Disclosure statement}

No author has any financial interest or received any financial benefit from this research.

\section{Conflict of interest}

The authors state no conflict of interest.

\section{References}

1. Hickson RC. Interference of strength development by simultaneously training for strength and endurance. Eur J Appl Physiol Occup Physiol. 1980;45(2-3):255263; doi: 10.1007/BF00421333.

2. Ross RE, Ratamess NA, Hoffman JR, Faigenbaum AD, Kang J, Chilakos A. The effects of treadmill sprint training and resistance training on maximal running velocity and power. J Strength Cond Res. 2009;23(2): 385-394; doi: 10.1519/JSC.0b013e3181964a7a.

3. Wilson JM, Marin PJ, Rhea MR, Wilson SMC, Loenneke JP, Anderson JC. Concurrent training: a metaanalysis examining interference of aerobic and resistance exercises. J Strength Cond Res. 2012;26(8):22932307; doi: 10.1519/JSC.0b013e31823a3e2d. 
A. Dalamitros et al., Intrasession resistance and sprint training in water polo

4. Docherty D, Sporer B. A proposed model for examining the interference phenomenon between concurrent aerobic and strength training. Sports Med. 2000;30(6): 385-394; doi: 10.2165/00007256-200030060-00001.

5. Sabag A, Najafi A, Michael S, Esgin T, Halaki M, Hackett D. The compatibility of concurrent high intensity interval training and resistance training for muscular strength and hypertrophy: a systematic review and meta-analysis. J Sports Sci. 2018;36(21):2472-2483; doi: 10.1080/02640414.2018.1464636.

6. Melchiorri G, Castagna C, Sorge R, Bonifazi M. Game activity and blood lactate in men's elite water-polo players. J Strength Cond Res. 2010;24(10):2647-2651; doi: 10.1519/JSC.0b013e3181e3486b.

7. Smith HK. Applied physiology of water polo. Sports Med. 1998;26(5):317-334; doi: 10.2165/00007256199826050-00003.

8. Sáez de Villarreal E, Suarez-Arrones L, Requena B, Haff GG, Ramos Veliz R. Enhancing performance in professional water polo players: dryland training, in-water training, and combined training. J Strength Cond Res. 2015;29(4):1089-1097; doi: 10.1519/JSC.000000 0000000707.

9. Botonis PG, Toubekis AG, Terzis GD, Geladas ND, Platanou TI. Effects of concurrent strength and highintensity interval training on fitness and match performance in water-polo players. J Hum Kinet. 2019;67: 175-184; doi: 10.2478/hukin-2019-0001.

10. Methenitis S. A brief review on concurrent training: from laboratory to the field. Sports. 2018;6(4):127; doi: 10.3390/ sports6040127.

11. Sousa AC, Marinho DA, Gil MH, Izquierdo M, Rodríguez-Rosell D, Neiva HP, et al. Concurrent training followed by detraining: does the resistance training intensity matter? J Strength Cond Res. 2018;32(3):632642; doi: 10.1519/JSC.0000000000002237.

12. Leveritt M, Abernethy PJ, Barry BK, Logan PA. Concurrent strength and endurance training. A review. Sports Med. 1999;28(6):413-427; doi: 10.2165/0000 7256-199928060-00004.

13. Doma K, Deakin GB, Bentley DJ. Implications of impaired endurance performance following single bouts of resistance training: an alternate concurrent training perspective. Sports Med. 2017;47(11):2187-2200; doi: 10.1007/s40279-017-0758-3.

14. Botonis PG, Toubekis AG, Platanou TI. Concurrent strength and interval endurance training in elite water polo players. J Strength Cond Res. 2016;30(1):126-133; doi: 10.1519/JSC.0000000000001091.

15. Meckel Y, Bishop D, Rabinovich M, Kaufman L, Nemet D, Eliakim A. Repeated sprint ability in elite water polo players and swimmers and its relationship to aerobic and anaerobic performance. J Sports Sci Med. 2013;12(4):738-743.

16. Girard O, Mendez-Villanueva A, Bishop D. Repeatedsprint ability - part I: factors contributing to fatigue.
Sports Med. 2011;41(8):673-694; doi: 10.2165/11590 550-000000000-00000.

17. Lisbôa FD, Raimundo JAG, Pereira GS, Ribeiro G, de Aguiar RA, Caputo F. Effects of time of day on race splits, kinematics, and blood lactate during a 50-m front crawl performance. J Strength Cond Res. 2021;35(3):819-825; doi: 10.1519/JSC.0000000000002794.

18. Rhea MR. Determining the magnitude of treatment effects in strength training research through the use of the effect size. J Strength Cond Res. 2004;18(4):918-920; doi: 10.1519/14403.1.

19. Botonis PG, Toubekis AG, Platanou TI. Evaluation of physical fitness in water polo players according to playing level and positional role. Sports. 2018;6(4):157; doi: 10.3390/sports6040157.

20. Hawley JA, Williams MM. Relationship between upper body anaerobic power and freestyle swimming performance. Int J Sports Med. 1991;12(1):1-5; doi: 10.1055/ s-2007-1024645.

21. Platanou T. Time-motion analysis of international-level water polo players. J Hum Mov Stud. 2004;46(4):319331.

22. Eddens L, van Someren K, Howatson G. The role of intra-session exercise sequence in the interference effect: a systematic review with meta-analysis. Sports Med. 2018;48(1):177-188; doi: 10.1007/s40279-017-0784-1. 